\title{
Experimental Evidence for Ultrafast Electronic Relaxation in Molecules, Mediated by Diffuse States
}

\author{
Eric Gloaguen, ${ }^{\dagger}$ Jean-Michel Mestdagh, ${ }^{\dagger}$ Lionel Poisson, ${ }^{\dagger}$ Fabien Lepetit, ${ }^{\dagger}$ \\ Jean-Paul Visticot, ${ }^{\dagger}$ Benoit Soep, ${ }^{\star}, \dagger$ Marcela Coroiu, ${ }^{\ddagger}$ André T. J. B. Eppink, ${ }^{\ddagger}$ and \\ David H.Parker $\ddagger$ \\ Contribution from the Laboratoire Francis Perrin, CNRS-URA-2453, DSM/DRECAM/Service \\ des Photons, Atomes et Molécules, C.E.A. Saclay, F-91191 Gif-sur-Yvette Cedex, France, and \\ Department of Molecular and Laser Physics, University of Nijmegen, Toernooiveld 1, \\ 6525ED Nijmegen, The Netherlands
}

Received April 8, 2005; E-mail: benoit.soep@cea.fr

\begin{abstract}
The existence of a mediating state for the ultrafast electronic relaxation in ethylenic-like molecules has been shown by femtosecond electron imaging techniques. This state is of Rydberg character, and its high efficiency is due to its ability to link the electron distributions of the initial and final electronic states.
\end{abstract}

\section{Introduction}

Molecules rarely reemit from the initially excited level after the absorption of a photon, and if the molecule is sufficiently complex, light emission, a slow process, is not the main decay channel for the excited state. Rapid photon energy relaxation without bond breaking is essential to maintaining life since our biological memory, DNA, is continuously exposed to light and photochemistry can be harmful to it. ${ }^{1,2}$ The electronic energy is rapidly degraded and thermalized within the ground electronic state of these systems, preventing most of the photochemistry by dissipation of the energy in the medium. These relaxation properties arise from coupling (essentially nonadiabatic) between the electronic configurations of the molecular systems accessible by optical excitation and the other energetically accessible configurations. However, in many molecules, this results in a nonradiative relaxation scheme akin to a tunneling effect that generates a fairly large decay time (picosecond time domain), allowing for photochemistry. Only recently, a direct mechanism has been observed experimentally ${ }^{3-8}$ which can allow an extremely fast relaxation in molecules; it involves a crossing between potential energy surfaces, ${ }^{9}$ named a conical intersection. This intersection or funnel connects directly the relevant excited

$\dagger$ CNRS.

$\doteqdot$ University of Nijmegen.

(1) Cohen, B.; Crespo-Hernández, C. E.; Kohler, B. Faraday Discuss. 2004, 127, 137-147.

(2) Nikogossian, D.; Angelov, D.; Soep, B.; Lindqvist, L. Chem. Phys. Lett. 1996, 252, 322

(3) Diau, E. W. G.; De-Feyter, S.; Zewail, A. H. J. Chem. Phys. 1999, 110, 9785-9788.

(4) Dongping, Z.; Diau, E. W. G.; Bernhardt, T. M.; De-Feyter, S.; Roberts, J. D.; Zewail, A. H. Chem. Phys. Lett. 1998, 298, 129-140.

(5) Mestdagh, J. M.; Visticot, J. P.; Elhanine, M.; Soep, B. J. Chem. Phys. 2000, 113, 237-248.

(6) Blanchet, V.; Zgierski, M.; Seideman, T.; Stolow, A. Nature 1999, 401, $52-54$.

(7) Farmanara, P.; Stert, V.; Radloff, W. Chem. Phys. Lett. 1998, 288, 518522

(8) Fuss, W.; Lochbrunner, S.; Muller, A. M.; Schikarski, T.; Schmid, W. E.; Trushin, S. A. Chem. Phys. 1998, 232, 161-174.

(9) Teller, E. J. Phys. Chem. 1937, 41, 109-116. states and provides a direct descent to the lowest energy surface, guided by the gradients along these surfaces. Nevertheless, discussions ${ }^{10}$ show that the passage through a conical intersection may be difficult in a complex system, where the descending wave packet can be reflected, lengthening the overall decay. In such case, the relaxation mechanisms can become slow in comparison to molecular movements, if the nonadiabatic coupling between the intersecting surfaces is not sufficient. Given the generally fast decay rates observed in a variety of molecules, and typically in ethylene-like compounds, ${ }^{5-8,11,12}$ it is conceivable that, besides the direct connection of the initial and final potential energy surfaces through a conical intersection, a complementary, general mechanism exists and increases the relaxation rate. We propose here that such a mechanism exists and involves mediating states or surfaces, allowing a more direct connection between the relevant excited surfaces. We have identified here these states in the case of a specifically selected ethylenic molecule.

Ethylene has long been recognized as displaying a conical intersection between the valence $\pi \pi^{*}$ state $\left(\mathrm{V}\right.$ of $\mathrm{C}^{\bullet}-\mathrm{C}^{\bullet}$ biradical-type and a zwitterionic state $\left(\mathrm{Z}, \mathrm{C}^{+}-\mathrm{C}^{-}\right) ;{ }^{13-15}$ these configurations were recognized early on as being accidentally degenerate in energy. ${ }^{16,17}$ The latter state $(Z)$ intersects with the ground state $(\mathrm{N})$ and allows dissipation of the electronic energy. The $\mathrm{V}-\mathrm{Z}$ intersection is caused by the different symmetry of the two states; in the planar configuration of ethylene, this is ${ }^{1} \mathrm{~B}_{1 \mathrm{u}}$ symmetry for the valence $\left(\pi \pi^{*}\right)$ and ${ }^{1} \mathrm{~A}_{\mathrm{g}}$ symmetry for the

(10) Jasper, A. W.; Zhu, C.; Nangia, S.; Truhlar, D. G. Faraday Discuss. 2004, 127, $1-22$.

(11) Pedersen, S.; Banares, L.; Zewail, A. H. J. Chem. Phys. 1992, 97, 88018804.

(12) Cyr, D. R.; Hayden, C. C. J. Chem. Phys. 1996, 104, 771-774.

(13) Ohmine, I. J. Chem. Phys. 1985, 83, 2348-2362.

(14) Ben Nun, M.; Martinez, T. J. Chem. Phys. 2000, 259, 237-248.

(15) Viel, A.; Krawczyk, R. P.; Manthe, U.; Domcke, W. J. Chem. Phys. 2004, 120, 11000-11010.

(16) Salem, L. Science 1976, 191, 822-830.

(17) Bonacic-Koutecký, V.; Bruckmann, P.; Hiberty, P.; Koutecký, J.; Leforestier, C.; Salem, L. Angew. Chem., Int. Ed. Engl. 1975, 14, 575-576. 


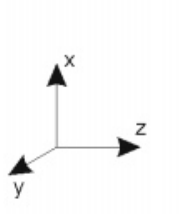

Planar $\left(\mathrm{D}_{2 \mathrm{~h}}\right)$

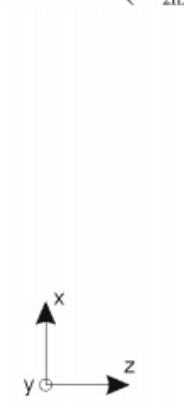

Twisted $\left(\mathrm{D}_{2}\right)$
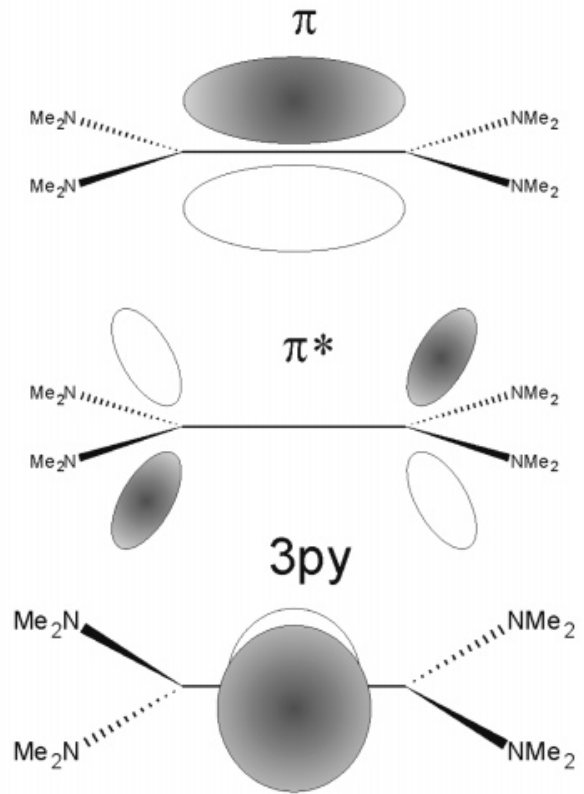

(A)

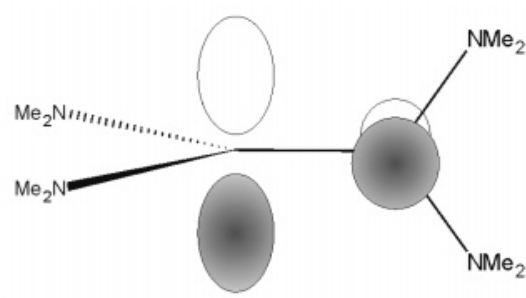

$90^{\circ}\left(\mathrm{D}_{2 \mathrm{~d}}\right)$

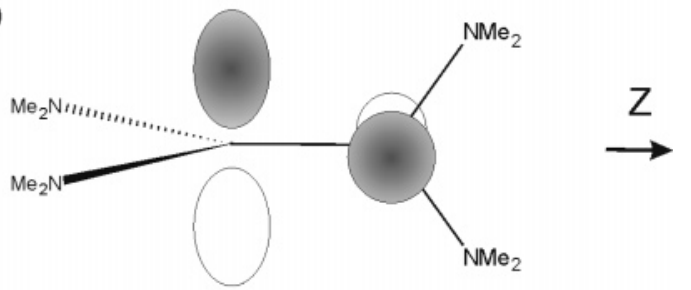

(B)

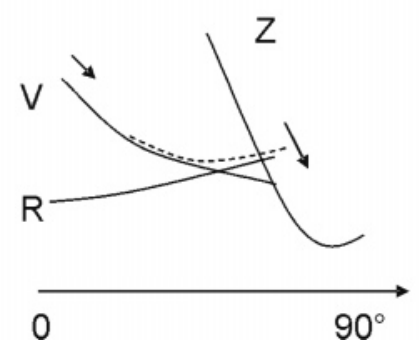

Figure 1. (A) Left, from top to bottom: in $D_{2 h}$ symmetry, the $\pi$ orbital and the $\pi^{*}$ orbital. After twisting the $\mathrm{C}=\mathrm{C}$ bond, in $D_{2}$ symmetry, the $3 \mathrm{p}_{y}$ Rydberg. The two electrons are schematically represented as occupying $\pi$ and $\pi^{*}$ orbitals that merge at the $90^{\circ}$ configuration into a degenerate representation $\mathrm{E}\left(D_{2 d}\right)$. The two electrons at this angle occupy separate p orbitals. (B) Schematic energy diagram.

$\left(\pi^{*}\right)^{2}$ configuration that correlates with the zwitterionic state. The active coordinates ${ }^{18}$ for this conical intersection are composed of the $\mathrm{C}-\mathrm{C}$ torsion coordinate (likely combined with the $\mathrm{C}-\mathrm{C}$ stretching ${ }^{15,19}$ ), upon which the two states intersect and the pyramidalization of the carbon atoms that couples the two states. Experimentally, several studies based on ion detection have shown evidence of ultrafast decay of excited ethylene and substituted ethylenes in the valence $\pi \pi^{*}$ state..$^{5-8,11}$ In the case of ethylene, the decay is as short as $\sim 30 \mathrm{fs},{ }^{5,7,20}$ but this could largely be due to the disappearance from the initial FranckCondon window. In fact, the overlap between the valence and zwitterionic electronic distributions is insufficient to allow for a strong coupling between the electronic configurations and to drive an extremely fast relaxation. Indeed, the excited ethylene molecule, after feeling a strong gradient along the $\mathrm{C}-\mathrm{C}$ torsion, should not experience an important gradient along the pyramidalization coordinate to reach the pyramidalized $\mathrm{Z}$ configuration. This configuration $\mathrm{Z}$ will localize two electrons on one carbon, which will be difficult to achieve due to the lack of overlap between the two perpendicular $\mathrm{p}$ orbitals, as pictured in Figure 1. This has been confirmed in recent calculations, where the gradient along the pyramidalization coordinate is found to be very small in the vicinity of the crossing, ${ }^{15}$ and it was also found by Martinez et al. ${ }^{14}$ that the population decay is longer than $100 \mathrm{fs}$, even though the deformation of ethylene only requires the displacement of the very light $\mathrm{H}$ atoms. Our claim in the present paper is that a mediating configuration with a large

(18) Bernardi, F.; Olivucci, M.; Robb, M. A. Chem. Soc. Rev. 1996, 25, 321328.

(19) Ben Nun, M.; Martinez, T. J. Chem. Phys. Lett. 1998, 298, 57-65.

(20) Stert, V.; Lippert, H.; Ritze, H.-H.; Radloff, W. Chem. Phys. Lett. 2004, 388, 144-149. spatial extension, namely a Rydberg state, may help link both configurations in $\mathrm{C}=\mathrm{C}$ double bond molecules, hence making the energy transfer ultrafast in situations where the group to be displaced is very heavy. This expectation arises from a longstanding puzzle concerning the ethylene molecule. An intersection of $\pi \pi^{*}$ and $3 p_{y}$ potential surfaces has been proposed, ${ }^{21}$ and Petrolongo et al. have given a detailed description. ${ }^{22}$ It was later realized that, in the case of ethylene, by comparison with the triplet ${ }^{3} \mathrm{~T} \pi \pi^{*}$ state, the Rydberg-valence coupling was overestimated. ${ }^{23-25}$ An exact calculation of the correlation interaction has distinguished these two states in ethylene despite their proximity in energy, ${ }^{24}$ More recently, the nonadiabatic coupling of the 3 s Rydberg state with the $\mathrm{V}$ state has also been proposed to be non-negligible. ${ }^{26}$ This question of Rydberg participation may seem controversial for ethylene, but not in heavier ethylenic compounds. Therefore, there is a need for experimental observations in cases where the Rydberg-valence interaction can be accounted for certain. In many ethylene-like molecules, both valence and zwitterionic states are relatively high in energy with respect to the ionization potential and overlap well with Rydberg states. We show here, for a specific example, evidence for a direct descent from the valence to a Rydberg surface(s), followed by evolution toward the zwitterionic state (Figure 1B).

(21) Buenker, R. J.; Peyerimhoff, S. D.; Hsu, H. L. Chem. Phys. Lett. 1971, 11, $65-70$.

(22) Petrongolo, C.; Buenker, R. J.; Peyerimhoff, S. D. J. Chem. Phys. 1982 $76,3655-3667$.

(23) Davidson, E. R. J. Phys. Chem. 1996, 100, 6161-6166.

(24) Muller, T.; Dallos, M.; Lischka, H. J. Chem. Phys. 1999, 110, 7176-7184.

(25) McMurchie, L. E.; Davidson, E. R. J. Chem. Phys. 1977, 66, 2959-2971.

(26) Baeck, K. K.; Martinez, T. J. Chem. Phys. Lett. 2003, 375, 299-308. 


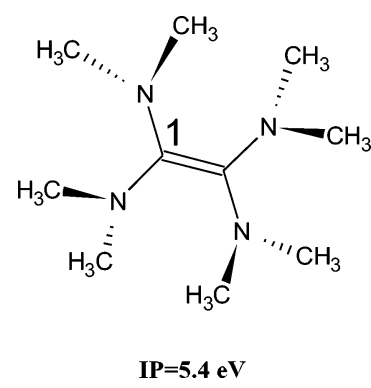

Figure 2. The TDMAE molecule.

We have chosen here as a case molecule the substituted ethylene, tetrakis(dimethylamino)ethylene, TDMAE, represented in Figure 2, which is known for its low ionization potential (5.4 $\mathrm{eV}$ ), due to the presence of the dimethylamino groups. ${ }^{27}$ TDMAE experiences a strong Rydberg-valence energy overlap since the valence region only decreases slightly in energy upon substitution, while Rydberg transitions shift with the ionization potential. TDMAE has been seeded in a supersonic helium beam and excited by a femtosecond laser (pump) in the UV at 266 $\mathrm{nm}(4.7 \mathrm{eV})$, which reaches the edge of the $\pi \pi^{*}$ valence region corresponding to the $\mathrm{C}=\mathrm{C}$ bond. The induced relaxation has been followed by a second femtosecond laser (either 1.5 or 3 $\mathrm{eV}$ ) that probes the temporal evolution by time-resolved photoelectron spectroscopy. The probe laser ionizes the TDMAE molecule, and the resulting photoelectron spectrum is a fingerprint of the evolution of its electronic configuration. ${ }^{6,28}$ For each traversed electronic state, there will be a different photoelectron spectrum indicative of the energy of the electronic state and its nature: valence, Rydberg, or zwitterionic.

The electron velocity imaging technique is perfectly adapted to femtosecond time-dependent photoelectron spectroscopy due to its very high sensitivity, where the electron counting rate is very high, similar to that for the corresponding ion. ${ }^{29}$ The energy resolution of the device is fully compatible with the frequency spread of $50 \mathrm{fs}$ Gaussian laser pulses, that is, $0.025 \mathrm{eV}$, and in addition, the technique yields another dimension, the angular distribution of the electrons with respect to the polarization direction of the ionizing laser field. This information on the angular distribution of the ejected electrons, although complex, since it depends on many parameters, can be essential in deciphering the evolution of the excited system through different electronic configurations.

\section{Results}

A typical image is shown in the right insert of Figure 3, where radial (electron energy) and angular (spatial anisotropy) dependences can be observed. The images are integrated over all angles, thus disregarding, in the first instance, the angular dependence to focus on their time dependence. The photoelectron spectra for various pump/probe delays can be combined, as shown in the left side of Figure 3. Four groups of bands can be seen, only one of which, $\mathrm{A}^{\prime}$, is weak; this one appears more distinctly in the magnification at the top of Figure 3. The first band, A, is close to the zero of energy of the electrons, with a

(27) Nakato, Y.; Ozaki, M.; Tsubomura, H. Bull. Chem. Soc. Jpn. 1972, 45, 1299-1305

(28) Tsubouchi, M.; Whitaker, B. J.; Wang, L.; Kohguchi, H.; Suzuki, T. Phys. Rev. Lett. 2001, 86, 4500-4503.

(29) Eppink, A. T. J. B.; Parker, D. H. Rev. Sci. Instrum. 1997, 68, 34773484 . shoulder extending to $0.6 \mathrm{eV}$. The cumulated energy of the pump and probe lasers is $6.2 \mathrm{eV}$, which allows disposal of a maximum $0.8 \mathrm{eV}$ for the electrons, using $5.4 \mathrm{eV}$ as the ionization potential of TDMAE. ${ }^{27}$ Therefore, any band beyond the shoulder of A will involve ionization by two probe photons, which is attainable given the intensity of the probe $\left(\sim 10^{11}\right.$ photons $\left./ \mathrm{cm}^{2}\right)$. The single photon character of band $A$ is verified by using a $400 \mathrm{~nm}$ probe (twice the energy of $800 \mathrm{~nm}$ ), in which case it is absent. In turn, bands $\mathrm{B}$ and $\mathrm{C}$ are still present, as displayed in Figure 4. The $\mathrm{A}^{\prime}$ band, located at $1.6 \mathrm{eV}$ as expected $(\mathrm{A},+1.5 \mathrm{eV})$ is more intense in this figure, due to a one-photon excitation.

These bands exhibit different time evolutions: peak A (maximum at $0.1 \mathrm{eV}$ ) can be simulated with an exponential decay of $200 \mathrm{fs}$, convoluted by the cross-correlation time of the lasers. We note that the shoulder has a shorter decay. The A band ends on a small (5\%) plateau. Peak B is simulated by an exponential rise of $200 \mathrm{fs}$, corresponding to the decay of A. The delayed appearance of this B band with respect to band A is clearly visible at $1 \mathrm{eV}$ in Figures 3 and 4. The decay of B is $380 \mathrm{fs}$ and is accompanied by the equivalent rise of $\mathrm{C}$ in 380 fs. The additional peak $\mathrm{A}^{\prime}$ (Figure 3 ) seen at $1.6 \mathrm{eV}$ mimics the evolution of peak A, the two-photon equivalent of $\mathrm{A}$. This is also verified in Figure 4, where $\mathrm{A}^{\prime}$ formed by a $3.1 \mathrm{eV}$ photon is more intense than when it is induced by two $1.55 \mathrm{eV}(800$ $\mathrm{nm})$ photons and shows the same sequential scheme. Band $\mathrm{A}^{\prime}$ appears first at $1.6 \mathrm{eV}$, decays into $\mathrm{B}$, which in turn forms $\mathrm{C}$. Measurements taken at longer times in the 266/800 nm scheme reveal that band $\mathrm{C}$ can be identified with a long-lived state with a $100 \mathrm{ps}$ lifetime, this is assigned to the zwitterionic state Z. ${ }^{30}$

The passage of $\mathrm{A}$, identified further as the initial $\pi \pi^{*}$ configuration, to C (zwitterionic) involves a short-lived step B, that is, a sequential decay. This reveals the existence of an intermediate between the initial state $\pi \pi^{*}$ and the $\mathrm{Z}$ state. Whether this pathway exists in ethylene is difficult to assess. In TDMAE, the bulkiness of the molecule and the inertia of the moving groups slow down all movements. Instead of $\mathrm{H}$ atoms, $\mathrm{N}\left(\mathrm{CH}_{3}\right)_{2}$ groups with a mass ratio of 44 (to $\mathrm{H}$ atoms) move. In addition, the energy profile of the intermediate step represented by band $\mathrm{B}$ is rather narrow $(\sim 0.2 \mathrm{eV})$, indicating a geometrical similarity with the observed ion; this can be recognized as a characteristic of a Rydberg state. We surmise (see below) that the corresponding state belongs to a Rydberg series converging to the adiabatic ionization potential at $5.4 \mathrm{eV}$. A width of $0.2 \mathrm{eV}$ is also found for band $\mathrm{C}$ (assigned in the following as $\mathrm{Z}$ ) and should be compared with that of band $\mathrm{A}$, which is $0.6 \mathrm{eV}$ broad, with sidebands. The assignment of these three bands $\mathrm{A}, \mathrm{B}$, and $\mathrm{C}$ to different electronic states (configurations) is further supported by polarization measurements, where the angular distribution of the electrons with respect to the ionization laser is recorded, as displayed in Figure 5. The polarization parameters $\beta$ extracted from these measurements represent the degree of alignment of the ejected electrons with respect to the probe ionization laser polarization direction. The anisotropy $\beta$ is obtained by a simple fitting of the angular dependence, shown in Figure 3, to the second order of the expansion $I(\theta)=C\left(1+\beta \times P_{2}(\cos \theta)+\ldots\right)$, where $P_{2}$ is a second-order Legendre polynomial. ${ }^{31}$ This is an approximation given the two-photon ionization process of the transient states,

(30) Soep, B.; Mestdagh, J. M.; Sorgues, S.; Visticot, J. P. Eur. Phys. J. D 2001, 14, 191-203.

(31) Yang, C. N. Phys. Rev. 1948, 74, 764-772. 


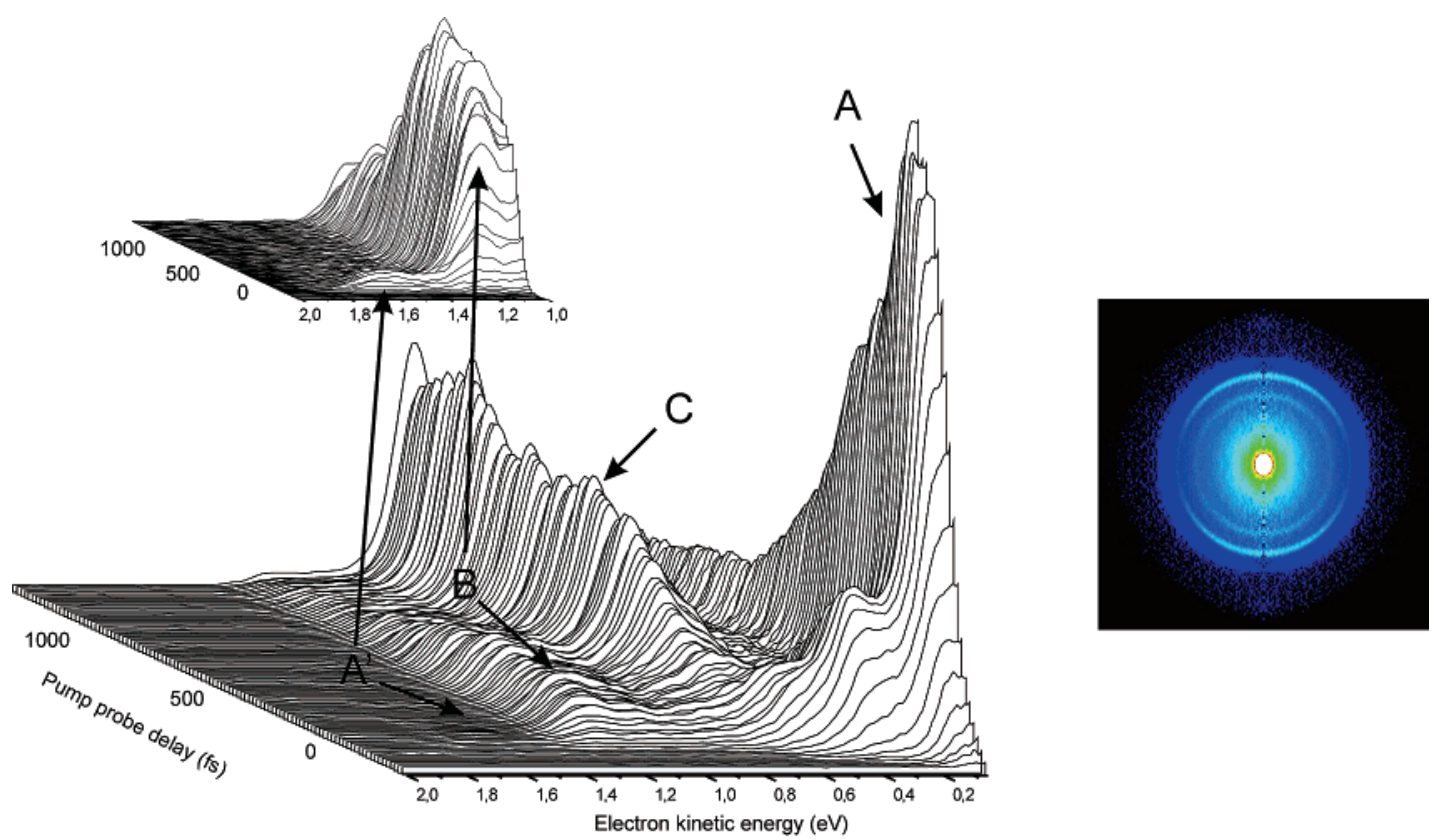

Figure 3. The time-resolved photoelectron spectra have been recorded in a special spectrometer with high collection efficiency, a velocity imaging device ${ }^{29}$ was used here for electrons. Right: electron image of TDMAE taken at $t>0$ of the correlation between the $266 \mathrm{~nm}$ (pump) and the $800 \mathrm{~nm}$ (probe) with perpendicular polarizations. The probe laser polarization is parallel to the plane of the detector. Left: $2 \pi$ angular integration of electron velocity images excited at $266 \mathrm{~nm}$ and ionized at $800 \mathrm{~nm}$. Each curve corresponds to a pump/probe time delay. The top insert shows a magnification of bands A' and B. The insert on the right is the complete electron velocity image, but integrated for all time delays.

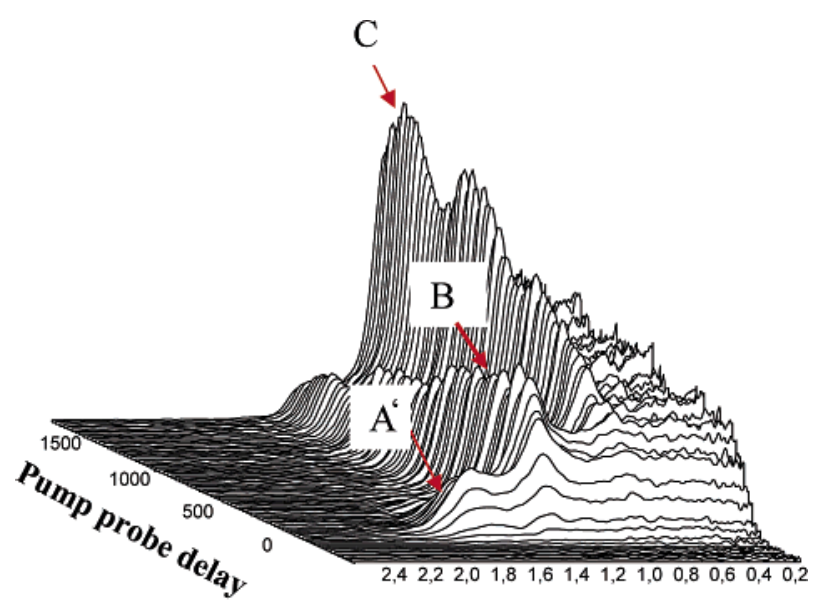

Electron energy $(\mathrm{eV})$

Figure 4. The time-resolved photoelectron spectra with angular integration of the electron velocity images of TDMAE excited at $266 \mathrm{~nm}$ and ionized at $400 \mathrm{~nm}$. Each curve corresponds to a pump/probe time delay.

but justified by the good agreement between the simulated angular distributions with $\beta_{2}$ and the experimental results. Beyond the $100 \mathrm{fs}$ duration of the pump beam, it appears in Figure 5 that the anisotropies associated with bands $\mathrm{B}$ and $\mathrm{C}$ show clearly constant values, $\beta_{2}=1.5$ and 0.9 , respectively, during the lifetime of the observed transient species. The high polarization observed for B is compatible with a Rydberg state assignment. Indeed, a maximum value $\beta=2$ can be obtained in the single-photon ionization of an atom through an $s-p$ transition. Molecular Rydberg states retain a strong asymptotic atomic character, thus the anisotropy of the ejected electron originating from a Rydberg orbital remains high. ${ }^{28}$ Unfortunately, molecular Rydberg states, especially the low-lying ones, are strongly influenced by the molecular core, as in our case ( $n$

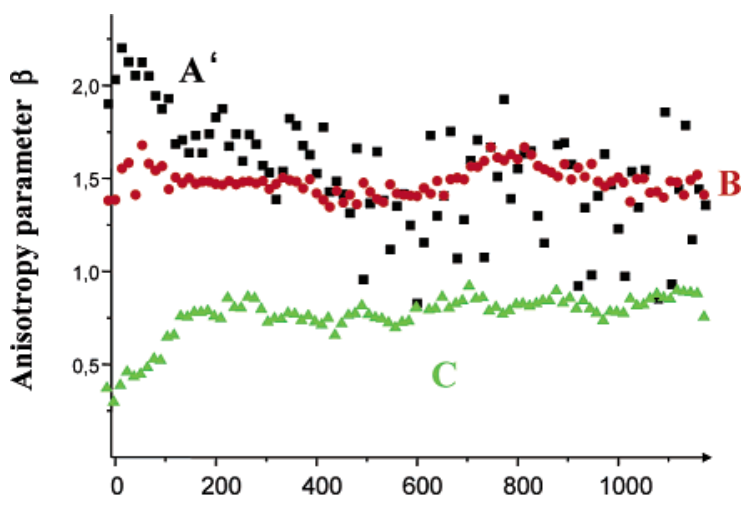

Pump-probe delay (fs)

Figure 5. Anisotropy parameter $\beta$ for each band as a function of the time delay between pump and probe. The two lasers have linear polarization, are parallel, and the polarization of the probe is in the plane of the imaging detector. The curves correspond to radial integration over 5 pixels for $\mathrm{B}-\mathrm{C}$ and 9 pixels for $\mathrm{A}^{\prime}$, centered at the kinetic energy maximum. This corresponds for peak $\mathrm{A}^{\prime}$ to a width of $0.2 \mathrm{eV}$ and $\sim 0.06 \mathrm{eV}$ for $\mathrm{B}$. The anisotropy was measured on peak $\mathrm{A}^{\prime}$, which is obtained with the same number of photons as B and $\mathrm{C}$.

$=3$ ). This produces a mixing of the partial electron waves, ${ }^{32}$ making the assignment of the ionized state purely from the angular distribution of the electrons difficult. Indeed, the angular distribution can be fitted simply with a second-order Legendre polynomial without an obvious fourth-order contribution, despite the two-photon ionization scheme at $800 \mathrm{~nm}$. Therefore, we retain only the high anisotropy as a character of the state. The $\mathrm{C}$ band that we assign to a zwitterionic state also keeps a diffuse character that may explain its anisotropy. However, it is difficult to assess by anisotropy measurements the nature of $\mathrm{A}(\beta=1.6$ past the initial $100 \mathrm{fs}$ ).

(32) Wang, K.; McKoy, V. Annu. Rev. Phys. Chem. 1995, 46, 275-304. 


\section{Discussion}

The present results have shown evidence of a sequential decay involving three states; this results from the fairly constant electron energies and anisotropies in the angular distribution. We have inferred that the intermediate state revealed by band $\mathrm{B}$ is of Rydberg nature, while $\mathrm{C}$ should correspond to another "local" state. This latter state corresponds to $\mathrm{Z}\left(\mathrm{C}^{+}-\mathrm{C}^{-}\right)$in Mulliken's description and decays in 100 ps to a charge-transfer state with a positive charge localized on the nitrogen atoms. ${ }^{30}$ As for band B, the width of the electron distribution and anisotropy points to a state akin to the ion; this also agrees with a zwitterionic character of $\mathrm{C}$.

The Rydberg character of the electronic configurations is the focus of this work and deserves discussion. The present molecule differs from ethylene by its low ionization potential due to the dimethylamino substitution. According to our present knowledge of the absorption spectrum of TDMAE coming from recent experiments in the gas phase and in rare gas matrices, ${ }^{33}$ and which differs slightly from the previously published gas-phase spectra, ${ }^{27,34,35}$ the electronic structure of TDMAE can be interpreted in the following way. The structure of TDMAE results in a red shift of the $\pi \pi^{*}$ transition to $266 \mathrm{~nm}$ in comparison with ethylene $(162 \mathrm{~nm})$, owing to a partial delocalization due to conjugation of the double bond with the lone pairs of the nitrogens. The absorption spectrum in TDMAE starts with diffuse Rydberg transitions at $400 \mathrm{~nm}$ (possibly 3s). ${ }^{27}$ The second region of absorption at $266 \mathrm{~nm}$ can be assigned by the matrix isolation spectra to a transition to a state whose valence character is dominant. This band is only slightly shifted from the gas to the matrix, and spectra taken in an argon matrix differ from that taken in a krypton matrix. The former matrix, argon, sweeps away to higher energies the low-lying Rydberg transitions at $400 \mathrm{~nm}$; these are maintained in the krypton matrix. Between these two matrices, the $266 \mathrm{~nm}$ band is unshifted. The absence of or very small shift in various condensed media is a criterion for the assignment of valence transitions. ${ }^{36}$

The valence $\pi \pi^{*}$ and Rydberg states are thus almost degenerate and most likely interacting in TDMAE, in contrast with ethylene when excited at $200 \mathrm{~nm}$. As shown by Davidson ${ }^{23}$ and Lischka, ${ }^{24}$ the $\mathrm{V}$ state of ethylene has a much smaller spatial extension $\left\langle x^{2}\right\rangle \sim 17 \AA^{2}$ than the $2^{1} \mathrm{~B}_{\mathrm{u}}$ Rydberg (90 $\AA^{2}$ ); hence, their coupling should be small due to a lack of overlap. For TDMAE, the situation is likely be different owing to the delocalization between the $\mathrm{C}=\mathrm{C}$ double bond and the nitrogens. This is manifested by two ionization limits, ${ }^{37} \pi-1$ and $n-1$ (N). Thus, the overlap should be more extensive in TDMAE as is the Rydberg-valence coupling. In addition, the size of the molecule points to a more extensive molecular character of the low-lying Rydberg states in TDMAE than in ethylene. This allows us to use here the picture developed originally for ethylene, where intense Rydberg-valence mixing was initially found in ab initio calculations. ${ }^{21,38}$ If we assume an ap-

(33) Gonzalez, A.; Gloaguen, E.; Soep, B.; Chergui, M. to be published, 2005. (34) Toby, S.; Astheimer, P. A.; Toby, F. S. J. Photochem. Photobiol., A 1992, $67,1-12$

(35) Holroyd, R. A.; Preses, J. M.; Woody, C. L.; Johnson, R. A. Nucl. Instrum. Methods Phys. Res., Sect. A 1987, A261, 440-444.

(36) Miron, E.; Raz, B.; Jortner, J. J. Chem. Phys. 1972, 56, 5265-5272.

(37) Sorgues, S.; Mestdagh, J. M.; Gloaguen, E.; Heninger, M.; Mestdagh, H.; Visticot, J. P.; Soep, B. J. Phys. Chem. A 2004, 108, 3884-3895.

(38) Salem, L. Electrons in Chemical Reactions: First Principles; John Wiley \& Sons: New York, 1982. proximately planar TDMAE molecule with $D_{2 h}$ symmetry, the $3 \mathrm{~d}_{x z}$ Rydberg state has a $\mathrm{B}_{2 \mathrm{~g}}$ symmetry, as does the $\pi^{*}$ orbital. The two likely mix, generating a diffuse orbital as proposed by Chupka et al. for ethylene. ${ }^{39}$ As the molecule twists along the $\mathrm{C}=\mathrm{C}$ bond, the symmetry is reduced to $\mathrm{D}_{2}$, the $\pi^{*}$ and the lower $3 \mathrm{p}_{y}$ Rydberg orbitals have the same $\mathrm{B}_{2}$ symmetry and can mix, yielding an avoided crossing. ${ }^{38}$ In this approximate symmetry, which supposes an initially planar molecule, there is no other Rydberg orbital of appropriate $\mathrm{B}_{2}$ symmetry involved with $n=$ 3 .

We can thus describe the initial step as a vertical excitation of a valence $\left(\pi \pi^{*}\right)$-Rydberg mixed state $\left(\pi 3 \mathrm{~d}_{x z}\right)$; this is observed through band A or $\mathrm{A}^{\prime}$. The configuration is mostly biradical $C^{\bullet}-C^{\bullet}$, but spatially more extended than a pure valence configuration. Quickly, upon twisting the former $\mathrm{C}=\mathrm{C}$ double bond, an intersection with a "purely" Rydberg potential surface sets in (presumably $\pi, 3 p_{y}$ ), as in (B). This corresponds to the passage of one of the electrons on the $3 p_{y}$ orbital. We surmise here that this $\mathrm{V}-\mathrm{R}$ intersection is very efficient and outreaches the $\mathrm{V}-\mathrm{Z}$ intersection; hence, the wave packet runs down this surface of $\mathrm{R}$ configuration. It is partly trapped, and once a strongly twisted geometry has been reached, the $3 p_{y}$ electron is easily transferred to the adjacent carbon, generating the $\mathrm{Z}$ configuration, which survives as band $\mathrm{C}$ for several 100 ps.

The present evolution results from fairly localized wave packet movements along several coordinates, two of which were already mentioned: the $\mathrm{C}-\mathrm{C}$ twist and the pyramidalization of carbon 1, as labeled in Figure 2. Wave packet movements are clear at initial times $(t<200 \mathrm{fs})$, as revealed by narrowing in time of the photoelectron spectrum of band A.

Thus, we have so far reported on the time evolution of the excited TDMAE molecule as implicitly consisting of a damped motion, where the wave packet created on the valence $\pi \pi^{*}$ surface could be rapidly spread over several coordinates of the potential surface after passage of the conical intersections. This spreading should result from the gradients on the multidimensional energy surface after a passage close to a conical intersection. Close inspection of Figures 3 and 4 reveals an apparently different situation, where peak $\mathrm{C}$ shows important modulations with a $500 \mathrm{fs}$ period that increases in amplitude as time goes by. Cuts of these bands have been made for bands $\mathrm{B}$ and $\mathrm{C}$ in Figure 6. They are well simulated using simple exponential evolution combined with an oscillatory behavior (see the Experimental Section). It is clearly evident that the oscillations increase within the 1200 fs time observation domain, and this is independent from the decay of the $\mathrm{B}$ and $\mathrm{C}$ bands. These oscillations are in phase in B and C, as shown in Figure 6. We, hence, infer that the observed vibrational coherence corresponds to a movement on a different coordinate than the "reaction coordinate". ${ }^{40,41}$ In the case where it would correspond to the reactive coordinate, it would be rapidly damped after the rise of B. Also, in the alternative case, where the system would be trapped along the reaction coordinate, we could expect a different phase for the old and the new configurations since these two could be detected at different times/locus on the

(39) Wiberg, K. B.; Hadad, C. M.; Foresman, J. B.; Chupka, W. A. J. Phys. Chem. 1992, 96, 10756-10768.

(40) Sorgues, S.; Mestdagh, J. M.; Visticot, J. P.; Soep, B. Phys. Rev. Lett. 2003, 91, 1-4.

(41) Trushin, S. A.; Yatsuhashi, T.; Fuss, W.; Schmid, W. E. Chem. Phys. Lett. 2003, 376, 282-291. 


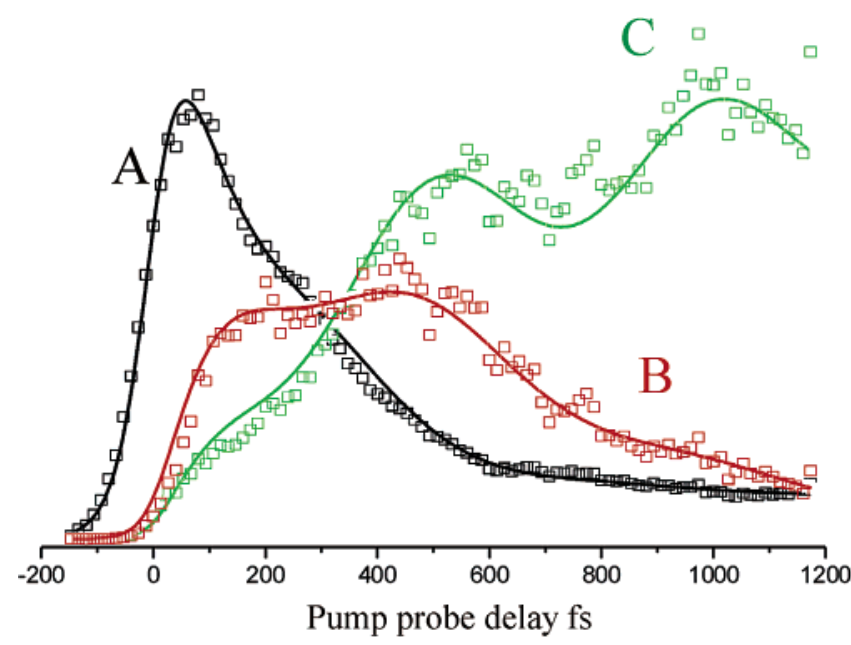

Figure 6. Time evolution of peaks A, B, and C, integrated over all angles and over 5 pixels radially, centered on the K.E. maximum. Peak B is magnified by a factor of 2 . The time evolution of these peaks has been simulated with a three state kinetic model (A, time constant $=200 \mathrm{fs} ; \mathrm{B}$, $380 \mathrm{fs} ; \mathrm{C}>10 \mathrm{ps}$, with a Gaussian convolution with $100 \mathrm{fs}$ fwhm), including a superimposed oscillatory behavior with a $500 \mathrm{fs}$ period.

potential surfaces. Such behavior has been observed in DABCO. ${ }^{42,43}$ The oscillatory movements for TDMAE occur then likely on perpendicular coordinates to the evolution of the decay which appears also due to the long coherence time for these oscillations. A full analysis of these wave packet movements will be the subject of a forthcoming paper.

\section{Conclusions}

Photoelectron imaging has revealed the existence of a shortlived (380 fs) intermediate state in the relaxation pathway of valence $\left(\pi \pi^{*}\right)$ excited TDMAE. This state is traversed by the wave packet descending from the excited region. It has been assigned to a Rydberg configuration and should be a key transient in the electronic relaxation mechanism of these ethylene-like molecules. The Rydberg intermediate provides, in the molecule that we have studied, TDMAE, sufficient electron density to accomplish the electron transfer to a zwitterionic configuration. The relaxation mechanism that we have proposed here is fairly general and should apply to many conjugated organic molecules of low ionization potential, that is, in molecules where substituents pull down Rydberg states into resonance with the $\pi \pi^{*}$ valence state. There, the relaxation of electronically excited states should often be facilitated by the passage through surfaces of diffuse electronic configuration, typically of Rydberg nature. We believe that the mechanism that we have brought into experimental evidence should apply in the relaxation of excited states of many biological systems, where crucial competition is at play between electronic relaxation and excited energy transfer or direct photochemistry.

\section{Experimental Section}

The experimental apparatus combines a pulsed supersonic beam with a velocity map imaging a Time-Of-Flight Mass-Spectrometer (TOFMS) device capable of imaging electrons and ions. ${ }^{29}$ The femtosecond laser source is the LUCA/SLIC femtosecond laser facility of Saclay was used to perform the pump (266 nm)-probe $(800 / 400 \mathrm{~nm})$ experiments.

(42) Hayden, C. C. In press.

(43) Seideman, T. Annu. Rev. Phys. Chem. 2002, 53, 41-65.
The beam is generated from a helium expansion passed over TDMAE at room temperature ( 0.3 Torr). The stagnation pressure $P_{0}$ is maintained low to avoid cluster formation, at 1 bar. The composition of the beam is verified by detecting TDMAE at mass 200. It is the only product in the $266 / 800 \mathrm{~nm}$ scheme, while a 185 fragment appears as a result of ion fragmentation with the $400 \mathrm{~nm}$ probe.

The pump laser at $266 \mathrm{~nm}$ performs a one-photon excitation of the TDMAE molecule, and the probe laser at $800 \mathrm{~nm}(400 \mathrm{~nm})$ achieves ionization. The power density of the probe is lower than $2 \times 10^{11}$ $\mathrm{W} / \mathrm{cm}^{2}$, enough to perform both one- and two-photon ionization of the electronically excited TDMAE. The cross-correlation widths of the lasers are $100 \mathrm{fs}(266 / 800 \mathrm{~nm})$ and $110 \mathrm{fs}(266 / 400 \mathrm{~nm})$, respectively.

Velocity Imaging. Ionization creates an expanding sphere of electrons which is projected on a position sensitive MCP. The emitted electrons excite a phosphor screen, and the image is captured by a CCD sensor. The raw images are recorded for each delay and stored after accumulation over several hundred laser shots. Electrons of increasing kinetic energies correspond to spheres of increasing sizes. The images have been transformed by the BASEX ${ }^{44}$ method to reconstruct the $3 \mathrm{D}$ features of the electron sphere. The images can be integrated angularly to derive the evolution of the photoelectron spectra as a function of time. In turn, the angular dependence with respect to the probe laser polarization can be fitted with a proper Legendre polynomial expansion and its time evolution analyzed.

Analysis of the Signals. The respective populations yielding the different electron signals are simulated using the following equations simulating a sequential decay $\mathrm{A} \rightarrow \mathrm{B} \rightarrow \mathrm{C} \rightarrow \mathrm{D}$. For $t>0$, the populations $P_{\mathrm{A}, \mathrm{B}, \mathrm{C}}(t)$ are with the time constants $\tau_{1,2,3}$ :

$$
\begin{gathered}
P_{\mathrm{A}}(t)=P_{\mathrm{A}}(0) \times e^{-t / \tau_{1}} \\
P_{\mathrm{B}}(t)=P_{\mathrm{A}}(0) \frac{\tau_{2}}{\tau_{1}-\tau_{2}}\left(e^{-t / \tau_{1}}-e^{-t / \tau_{2}}\right) \\
P_{\mathrm{C}}(t)=P_{\mathrm{A}}(0)\left(\frac{\tau_{1} \tau_{3}}{\left(\tau_{1}-\tau_{2}\right)\left(\tau_{1}-\tau_{3}\right)} e^{-t / \tau_{1}}-\frac{\tau_{2} \tau_{3}}{\left(\tau_{1}-\tau_{2}\right)\left(\tau_{2}-\tau_{3}\right)} e^{-t / \tau_{2}}+\right. \\
\left.\frac{\tau_{3}^{2}}{\left(\tau_{1}-\tau_{3}\right)\left(\tau_{2}-\tau_{3}\right)} \tau e^{-t / \tau_{3}}\right)
\end{gathered}
$$

These populations are multiplied by a $\sin ^{2}(2 \pi t / T)$, where $T$ is the period of oscillation. The resulting signal is convoluted with a hyperbolic secant function representing the laser response $F(t)=1 / c^{2}\left(t / L_{\text {laser }}\right)$, where $L_{\text {laser }}$ is the cross-correlation width of the lasers.

Acknowledgment. The authors thank O. Gobert, P. Meynadier, and M. Perdrix, who are responsible for developing, maintaining, and running the femtosecond laser facility LUCA (Laser Ultra-Court Accordable) of the CEA, DSM/DRECAM. This work is partly supported by the European Community through the PICNIC network (Product Imaging and Correlation: Non-adiabatic Interactions in Chemistry) under Contract Number HPRN-CT-2002-00183. D.H.P., M.A.C., and A.E. are also grateful to the European Union for support to access the SLIC/LASERLAB ultrafast laser facility at CEA, Saclay. D.H.P. and M.A.C. acknowledge support by the Dutch National Science Foundation, CW-NWO.

\section{JA052269U}

(44) Dribinski, V.; Ossadtchi, A.; Mandelshtam, V. A.; Reisler, H. Rev. Sci. Instrum. 2002, 73, 2634-2642. 